\title{
Hypocoagulability in Traumatic Brain Injury as Measured by Traditional Means and Thrombelastography
}

\author{
Sherry L Sixta ${ }^{1}$, Jessica C Cardenas ${ }^{1,3}$, Ryan Kitagawa ${ }^{2}$, Charles E Wade ${ }^{1,3}$, John B Holcomb ${ }^{1,2}$ and Bryan A Cotton ${ }^{1,2 *}$ \\ ${ }^{1}$ Departments of Surgery, The University of Texas Health Science Center at Houston, TX, USA \\ ${ }^{2}$ Neurosurgery, The University of Texas Health Science Center at Houston, TX, USA \\ ${ }^{3}$ The Center for Translational Injury Research, The University of Texas Health Science Center at Houston, TX, USA
}

\begin{abstract}
Background: Conflicting data exist regarding the association of traumatic brain injury (TBI) with coagulopathy as measured by conventional coagulation testing (CCT).
\end{abstract}

Objective: This study is intended to determine the prevalence of coagulopathy in TBI patients by CCT and rapid thrombelastography ( $r-T E G)$ and to determine the relationship between these laboratory tests and mortality.

Methods: Over an 18-month period, the admission r-TEG values (ACT, R-value, k-time, alpha, mA, and LY30) and CCTs (aPTT, INR, platelet count, and fibrinogen) were recorded on the highest-level trauma activations admitted directly from the injury scene. Patients were then categorized as non-TBI (Head AIS 0-2) or isolated TBI (Head AIS >2 and all other AIS scores $\leq 2$ ), and all other patients were excluded. A patient has determined to have a coagulopathy if one or more of the following was present: aPTT $>35 \mathrm{sec}$, INR $>1.5$, platelet count $<150$, fibrinogen $<180, A C T>128$, $R>1.1, k>2.5$, alpha $<56, m A<55, L Y 30>3 \%$.

Results: 1847 non-TBI patients and 77 isolated-TBI were identified. No significant difference was detected between the groups by CCT, but the isolated-TBI group was found to have more patients with $m A<55$ by $r$-TEG $(p=0.034)$. Multiple logistic regression revealed $m A<55(O R 3.68 ; p=0.015)$ or alpha $<56(O R 22.23 ; p<0.001)$ were predictive of 24-hour mortality in all patients. However, in isolated TBI patients, the presence of either of these hypocoagulable variables was uniformly fatal.

Conclusion: No difference in coagulopathy prevalence was observed between TBI and non-TBI patients by CCT, but a higher prevalence of coagulopathy by r-TEG values was found in isolated TBI patients and was predictive of mortality.

Keywords: Coagulation; Fibrinogen; Mortality; Platelets; Prothrombin time; Thrombelastography; Traumatic brain injury

\section{Introduction}

Traumatic brain injury (TBI) is the leading cause of mortality in trauma patients and accounts for up to $60 \%$ of traumatic deaths $[1,2]$. Coagulopathy frequently accompanies severe TBI and compounds the risk of morbidity and mortality [3-9]. However, the incidence and factors associated with coagulopathy in TBI has not been firmly established $[4,7,10-12]$. In 2009, Talving et al published the only prospective data to date from a level I urban trauma center, demonstrating a $34-36 \%$ incidence of coagulopathy in TBI patients, as measured by International Normalized Ration (INR), activated partial thromboplastin time (aPTT), and platelet count. In this study, independently associated risk factors for coagulopathy among isolated TBI patients included a GCS $\leq 8$, an ISS $\geq 16$, the presence of cerebral edema, subarachnoid hemorrhage, or a midline shift upon admission [4].

Conventional coagulation tests (CCTs) such as prothrombin time (PT), aPTT, INR, platelet counts, and fibrinogen levels have been used to diagnose and treat coagulopathy in TBI patients. However, CCTs illustrate the coagulation process in a static state, and the acceptable levels for CCTs in TBI are largely center dependent and have not been validated for use in the characterization of coagulopathy during active hemorrhage $[13,14]$. Additionally, long turn-around times make CCTs less than ideal for trauma and TBI patients $[15,16]$.

Thrombelastography (TEG) was introduced in 1948 as a device that kinetically measures the viscoelastic properties of a blood sample as it clots following kaolin-induced activation via a representative tracing [17]. This tracing or "thromboelastogram" extrapolates and renders numerical data that quantifies the functionality of plasma proteins, platelets, and fibrinogen as well as platelet interaction with fibrinogen and fibrinolysis [18]. TEG allows for a comprehensive evaluation of the clotting cascade in an expedited fashion for diagnostic purposes and therapeutic interventions $[19,20]$.

Rapid TEG (r-TEG) is an accelerated version of thrombelastography that utilizes tissue factor, in addition to the kaolin, to expedite clotting cascade activation and to provide fast and reliable coagulation profiles in emergency settings [15]. Cotton et al and Holcomb et al demonstrated that the r-TEG values for activated clotting time (ACT), k-time, and the R-time were available within 5 minutes, and the maximal amplitude $(\mathrm{mA})$ and alpha angle were available within 15 minutes. These values correlated with CCTs in relation to coagulopathy $[16,21]$. To date, few reports have described the utility of $r$-TEG in diagnosing coagulopathy in TBI patients. The purpose of this study was to determine the prevalence of coagulopathy in TBI by admission r-TEG and CCT variables and to define the association between $r$-TEG hypocoagulability and mortality in this population.

${ }^{*}$ Corresponding author: Bryan A Cotton, Professor of Surgery, Department of Surgery and the Center for Translational Injury Research, University of Texas Health Science Center, Houston, TX, USA. Tel: 713-500-7354; Fax: 713-512-7135 E-mail: Bryan.A.Cotton@uth.tmc.edu

Received September 04, 2015; Accepted September 15, 2015; Published September 22, 2015

Citation: Sixta SL, Cardenas JC, Kitagawa R, Wade CE, Holcomb JB, et al (2015) Hypocoagulability in Traumatic Brain Injury as Measured by Traditional Means and Thrombelastography. J Neurol Neurophysiol 6: 316. doi:10.4172/21559562.1000316

Copyright: $\odot 2015$ Sixta SL, et al. This is an open-access article distributed under the terms of the Creative Commons Attribution License, which permits unrestricted use, distribution, and reproduction in any medium, provided the original author and source are credited. 


\section{Materials and Methods}

\section{Study setting}

This study was performed with approval from the University of Texas Health Science Center-Houston and the Memorial Hermann Hospital Institutional Review Boards. The Texas Trauma Institute at Memorial Hermann Hospital is an American College of Surgeons verified Level I trauma center that admits over 6,000 trauma patients annually.

\section{Selection of participants}

Using the institution's Trauma Registry of the American College of Surgeons database, we evaluated all adult trauma patients admitted between September 2009 and February 2011 who were the institution's highest-level trauma activation and were transported directly from the scene. Patients younger than 18 years, those with burn wounds greater than $20 \%$ total body surface area, or those who died within 30 minutes of arrival were excluded.

\section{Laboratory setting and processing of specimens}

All admission $r$-TEG specimens were processed on a TEG thrombelastography 5000 (Hemoscope Corporation, Niles, IL). Blood specimens for $\mathrm{r}$-TEG were acquired during the primary or secondary survey evaluation of all major trauma activations and performed utilizing a previously described rapid citrate "reversal" method $[16,18]$. In addition, conventional coagulation tests (CCTs) were obtained and included aPTT, INR, platelet counts, and fibrinogen levels.

\section{Measurements}

The r-TEG's ACT (normal range, 86-118 seconds) is calculated from the $\mathrm{R}$ time, which is the time between clot activation and when the waveform reaches $2-\mathrm{mm}$. The ACT $[(1.5592 * \mathrm{R})+50.48]$ is prolonged with factor deficiency or enzymatic hypocoagulable states. The k-time (clot kinetics) reflects the kinetics of the clot's formation and is the time in minutes required to reach $20-\mathrm{mm}$ clot strength. The k-time's normal range is $1-2$ minutes and can be prolonged with coagulation enzyme deficiencies or hypofibrinogenemia. The alpha angle (normal range, 66-82 degrees) is the slope of the tracing that represents the rate of clot formation and is also decreased with hypofibrinogenemia. The $\mathrm{mA}$ (normal range, $54-72 \mathrm{~mm}$ ) is the maximum amplitude of the tracing and reflects platelet and fibrinogen contributions to clot strength. Low $\mathrm{mA}$ values are indicative of low platelet counts or platelet inhibition. LY30 (normal range, 0.0-7.5\%) represents the percent amplitude reduction at 30 minutes after $\mathrm{mA}$ and reflects the degree of fibrinolysis.

\section{Definitions and outcomes}

To assess the impact of isolated TBI (rather than concomitant injuries) on the development of coagulopathy, non-TBI was defined a head abbreviated injury scale (AIS) $\leq 2$, and isolated TBI was defined as having head AIS $>2$ and all other body system AIS $\leq 2$. The presence/ severity of TBI was confirmed by patient chart review.

Coagulopathy was defined by the presence of one or more of the following: aPTT $>35$ seconds, INR $>1.5$, platelet count $<150 \times 10^{9} / \mathrm{L}$, fibrinogen $<180 \mathrm{mg} / \mathrm{dL}$, ACT $>128$ seconds, $\mathrm{k}>2.5$ minutes, alpha $<56$ degrees, $\mathrm{mA}<55$ millimeters, and LY30 >3\% (21). These critical TEG values were based on our previous work where inflection points on scatter-plot analyses identified when transfusion requirements and mortality increased substantially. Multivariate models also demonstrated that these values accurately predict the risks of both massive transfusion and mortality in critically ill patients [18].

\section{Statistical analysis}

Continuous data were presented as medians with $25^{\text {th }}$ and $75^{\text {th }}$ interquartile range with comparisons between groups performed using the Wilcoxon rank sum (Mann-Whitney U test). Categorical data were reported as proportions and, where appropriate, tested for significance using chi-square or Fisher exact tests. The primary data analysis evaluated each admission lab value between those with isolated TBI and those without TBI. The two groups were then evaluated for their respective 24-hour and 30-day mortality and the impact of hypocoagulability on outcomes. Purposeful regression modeling was then used to construct a multivariate logistic regression model evaluating 24-hour and 30-day mortality. This was done using the technique of purposeful selection of covariates described by Hosmer and Lemeshow [22]. This was first conducted on the population as a whole, then on the isolated TBI patients only. In an effort to minimize the risk of falsely identifying significant results with multiple comparisons, all variables were pre-specified and judged a priori to be clinically relevant. These independent variables included age, gender, race, ISS, emergency department vitals and laboratory values, and prehospital and hospital fluid administration and transfusions. The variables were then entered into step-wise regression that generated three variables of significance (GCS, SBP and ISS), which were then applied to a multivariate logistic regression analysis along with the presence of hypocoagulability, defined by admission $\mathrm{mA}$ and alpha values. All statistical tests were two tailed with $\mathrm{p}<0.05$ declared significant. STATA Statistical software (version 12.0; College Station, TX) was used for analysis.

\section{Results}

\section{Admission coagulation profiles and prevalence of coagulopathy}

2069 patients were initially identified, but 145 were excluded because they had both head and at least one other body region AIS score $>2$. Of the 1924 remaining patients, 1847 were in the non-TBI group and 77 were in the isolated TBI group (Table 1). No observed differences between the group in age, race, gender, admission blood alcohol level ( $\mathrm{p}=0.616)$, base deficit $(\mathrm{p}=0.580)$, lactate $(\mathrm{p}=0.416)$ or hemoglobin $(\mathrm{p}=0.344)$. Isolated TBI patients had both lower arrival GCS $(\mathrm{p}<0.001)$ and higher ISS scores $(\mathrm{p}<0.001)$. A slightly prolonged INR was noted in the isolated TBI group $(\mathrm{p}=0.013)$, however no significant differences were observed in admission $r$-TEG values.

The two groups were then assessed by arrival CCT and r-TEG values, dichotomized along previously described hypocoagulable values (Table 2) [21]. A higher prevalence of coagulopathy by $r$-TEG alpha $(\mathrm{p}=0.001)$ and $\mathrm{mA}$ was observed in isolated TBI patients $(\mathrm{p}=0.039)$, however no difference in the prevalence of coagulopathy by CCTs values was detected. While 24-hour mortality was similar for all patients $(6.3 \%$ vs. $5.7 \%, p=0.869)$, univariate analysis demonstrated higher 30-day mortality in the isolated TBI group compared to nonTBI patients $(20.7 \%$ vs. $11.1 \%, \mathrm{p}<0.001)$.

\section{Association between r-TEG variables and mortality}

Our stepwise regression model generated three variables of significance: arrival GCS, systolic blood pressure and ISS. These variables were then entered into our purposeful regression model along with hypocoagulable CCT, and r-TEG values. After controlling for the three variables of significance, none of the hypocoagulable CCT variables were associated with increased 24-hour or 30-day mortality, but both r-TEG mA (OR 3.68, 95\% C.I. 1.29-10.51, p=0.015) and alpha- 
Citation: Sixta SL, Cardenas JC, Kitagawa R, Wade CE, Holcomb JB, et al. (2015) Hypocoagulability in Traumatic Brain Injury as Measured by Traditional Means and Thrombelastography. J Neurol Neurophysiol 6: 316. doi:10.4172/2155-9562.1000316

\begin{tabular}{|l|c|c|c|}
\hline & $\begin{array}{c}\text { Non-TBI } \\
(\mathrm{n}=1847)\end{array}$ & Isolated-TBI $(\mathrm{n}=77)$ & $\mathrm{p}$-value \\
\hline Median age, years & $33(23,49)$ & $31(23,58)$ & 0.739 \\
\hline Male gender, $\%$ & $75 \%$ & $74 \%$ & 0.807 \\
\hline White race, $\%$ & $54 \%$ & $57 \%$ & 0.531 \\
\hline Blunt mechanism, \% & $71 \%$ & $80 \%$ & 0.073 \\
\hline Median arrival GCS & $14(3,15)$ & $3(3,7)$ & $<0.001$ \\
\hline Median arrival SBP, mmHg & $130(107,149)$ & $133(118,152)$ & 0.063 \\
\hline Median arrival HR, bpm & $97(80,117)$ & $93(81,110)$ & 0.306 \\
\hline Median ISS & $16(18,25)$ & $24(16,29)$ & $<0.001$ \\
\hline Median platelet count $\times 10 \% / L$ & $240(193,292)$ & $231(206,299)$ & 0.796 \\
\hline Median aPTT, sec & $27.4(24.7,30.4)$ & $27.2(24.7,29.5)$ & 0.866 \\
\hline Median INR & $1.10(1.02,1.22)$ & $1.16(1.08,1.34)$ & 0.013 \\
\hline Median fibrinogen, mg/dL & $274(224,329)$ & $232(183,322)$ & 0.146 \\
\hline Median r-TEG ACT, sec & $113(105,128)$ & $121(105,128)$ & 0.395 \\
\hline Median r-TEG k-time, min & $1.4(1.1,1.8)$ & $1.6(1.2,1.9)$ & 0.117 \\
\hline Median r-TEG alpha, deg & $73(69,76)$ & $71(63,74)$ & 0.059 \\
\hline Median r-TEG mA, mm & $64(59,68)$ & $64(58,66)$ & 0.139 \\
\hline Median r-TEG LY30, \% & $0.7(0.1,1.8)$ & $0.7(0.0,1.6)$ & 0.230 \\
\hline
\end{tabular}

All continuous values are expressed in medians with interquartile range $\left(25^{\text {th }}\right.$ $75^{\text {th }}$ ); TBI: traumatic brain injury; GCS: Glasgow coma scale; SBP: systolic blood pressure; mmHg: millimeters mercury; HR: heart rate; bpm: beats per minute; ISS: injury severity score; aPTT: activated partial thromboplastin time; INR: international normalized ratio; L: liter; sec: seconds; mg/dL: milligrams per deciliter; $r$-TEG: rapid thrombelastography; ACT: activated clotting time; mA: maximal amplitude; LY30: lysis 30 minutes after $\mathrm{mA}$ achieved; min: minutes; deg: degrees

Table 1: Comparison of demographics, admission physiology and lab values and injury severity among isolated TBI vs. non-TBI patients.

\begin{tabular}{|c|c|c|c|}
\hline & $\begin{array}{c}\text { Non-TBI } \\
(\mathrm{n}=1847)\end{array}$ & $\begin{array}{c}\text { Isolated-TBI } \\
(\mathrm{n}=77)\end{array}$ & p-value \\
\hline aPTT $>35 \mathrm{sec}$ & $7 \%$ & $8 \%$ & 0.793 \\
\hline INR>1.5 & $8 \%$ & $6 \%$ & 0.538 \\
\hline Platelet count $<150 \times 10 \% / \mathrm{L}$ & $7 \%$ & $11 \%$ & 0.259 \\
\hline Fibrinogen $<180 \mathrm{mg} / \mathrm{dL}$ & $3 \%$ & $6 \%$ & 0.163 \\
\hline r-TEG ACT $>128 \mathrm{sec}$ & $14 \%$ & $19 \%$ & 0.306 \\
\hline r-TEG $k$-time $>2.5 \mathrm{~min}$ & $9 \%$ & $8 \%$ & 0.452 \\
\hline r-TEG alpha angle $<56 \mathrm{deg}$ & $1 \%$ & $5 \%$ & 0.001 \\
\hline r-TEG $\mathrm{mA}<55 \mathrm{~mm}$ & $7 \%$ & $10 \%$ & 0.039 \\
\hline r-TEG LY30 >3\% & $9 \%$ & $6 \%$ & 0.837 \\
\hline
\end{tabular}

TBI: traumatic brain injury; aPTT: activated partial thromboplastin time; INR: international normalized ratio; $\mathrm{ACT}$ : activated clotting time; $\mathrm{mA}$ : maximal amplitude; LY30: lysis 30 minutes after $\mathrm{mA}$ achieved

Table 2: Comparison of the prevalence of admission hypocoagulability by CCTs and $r$-TEG between non-TBI and isolated TBI patients.

\begin{tabular}{|c|c|c|c|}
\hline & Odds ratio & $95 \%$ C.I. & p-value \\
\hline Hypocoagulability by $\mathrm{mA}$ & 3.35 & $2.26-4.91$ & $<0.001$ \\
\hline Hypocoagulability by alpha & 6.89 & $3.71-12.63$ & $<0.001$ \\
\hline Arrival GCS & 0.92 & $0.85-1.004$ & 0.063 \\
\hline Arrival SBP & 1.01 & $0.99-1.02$ & 0.198 \\
\hline ISS & 1.07 & $1.02-1.11$ & 0.003 \\
\hline
\end{tabular}

GCS: Glasgow coma scale; SBP: systolic blood pressure; ISS: injury severity score; 95\% C.I.: $95^{\text {th }}$ percent confidence interval; $\mathrm{mA}$ : maximal amplitude

Table 3: Exact logistic regression modeling for 30-day mortality in the entire population $(n=1924)$.

angle (OR 22.23, 95\% C.I. 6.32-78.28, p<0.001) were independent predictors of 24-hour mortality.

Fisher's exact logistic regression revealed that r-TEG markers of hypocoagulability were independently predictive of 30-day mortality in all patients ( $\mathrm{mA} \mathrm{OR} 3.35,95 \%$ C.I. 2.26-4.91 and alpha-angle OR 6.89, 95\% C.I. 3.71-12.63) (Table 3). This analysis was then repeated evaluating only those patients with isolated TBI. After controlling for arrival blood pressure, GCS and ISS, none of the hypocoagulable CCT values were able to predict an increased risk of 24-hour or 30-day mortality. However, all isolated TBI patients who were hypocoagulable by admission r-TEG (mA or alpha-angle) died within 24-hours.

\section{Discussion}

Similar to the acute coagulopathy of trauma (ACoT), TBI coagulopathy has been associated with variations in local and systemic coagulation processes including both hypo- and hypercoagulability. A variety of mechanisms have been hypothesized including alterations in the release of tissue factor, platelet dysfunction, consumptive coagulopathy, and the activation of protein $\mathrm{C}$ pathways secondary to hypoperfusion [12,23-31].

Abnormalities in admission PT, aPTT, INR, and fibrinogen degradation products have all been linked to increased morbidity and mortality in TBI patients [3,4,7,12,27,28,32-37]. However, when we analyzed admission CCT values on 1924 of our highest-level trauma activations over 18 months, no statistically significant difference in the prevalence of coagulopathy based on these values was detected in TBI patients compared to non-TBI patients (Table 2). This observation brings into question the utility of conventional laboratory tests in identifying coagulopathy in this patient population.

In recent years, TEG has improved characterization, identification, and treatment $\mathrm{ACoT}[16,18,21,38,39]$ but there is a paucity of literature analyzing coagulopathy associated with TBI by TEG. A thromboelastogram is a graphic display of the viscoelastic properties of the clotting process of a whole blood specimen as it transitions through the clotting process. It includes the initial enzymatic activation of coagulation, the interactions of fibrinogen and plasma proteins with platelets to form the fibrin clot, and the eventual clot dissolution [40]. Each variable characterizes the dynamic alterations between hyper and hypocoagulability, making it an additional tool to diagnose and treat TBI coagulopathy [41].

When evaluating the prevalence of coagulopathy between isolated TBI and non-TBI patients by CCT and r-TEG, we found that isolated TBI patients were more likely to be coagulopathic by $r$-TEG alpha angle and $\mathrm{mA}$ values but not by CCT. When multiple logistic regression was applied to analyze 24-hour mortality, CCTs were not predictive, but both $\mathrm{r}$-TEG $\mathrm{mA}<55$ and alpha-angle $<56$ were independent predictors of 24 hour and 30-day mortality in all patients. Hypocoagulability by $\mathrm{mA}$ and by alpha-angle had a 3 fold and 7 fold increased risk of 30day mortality respectively (Table 3 ). When the isolated-TBI group was analyzed alone, we found hypocoagulability by either r-TEG $\mathrm{mA}$ or alpha-angle to be uniformly fatal within 24 hours whereas CCT values had no association with 24 -hour or 30 -day mortality in this population.

Previous authors have also reported that platelet dysfunction in TBI patients correlates with poor outcomes, $[12,37]$ but to our knowledge, no other publications correlate TBI coagulopathy with $\mathrm{mA}$ or alpha-angle TEG values. Kunio et al analyzed 69 polytrauma patients with TBI and found that these patients had an increased R-time on traditional TEG [8]. The R-time is comparable to the ACT on r-TEG, which signifies a prolongation of the time to fibrin clot formation due to clotting factor deficiency.

The $\mathrm{mA}$, or maximum amplitude, on the TEG represents clot strength and is dependent upon platelet function, concentration, 
Citation: Sixta SL, Cardenas JC, Kitagawa R, Wade CE, Holcomb JB, et al. (2015) Hypocoagulability in Traumatic Brain Injury as Measured by Traditional Means and Thrombelastography. J Neurol Neurophysiol 6: 316. doi:10.4172/2155-9562.1000316

and interaction with fibrinogen. The alpha-angle measures the rate of fibrin clot formation and reflects the interaction of platelets and clotting factors. Our data indicate that TBI coagulopathy is reflected in these values and contingent upon platelet and fibrinogen interaction. However, mechanistic studies are needed to validate these assumptions and further define the contribution of platelet function and their interactions with fibrinogen to coagulopathy in TBI patients.

Currently, when CCTs in the TBI population are found to be within normal limits, no interventions are initiated. However, transfusions of platelets and concentrated clotting factors such as cryoprecipitate or fibrinogen concentrates based on the r-TEG results may improve the outcome in this population. Further prospective studies are necessary to validate our findings and to test the outcomes of these interventions.

To our knowledge, this is the largest study analyzing coagulopathy in TBI patients by TEG, but several limitations exist. Although the data was prospectively gathered, it was retrospectively analyzed. Additionally, this analysis is based upon admission values obtained from a single center and included only the highest level of trauma activations. We also excluded 143 polytrauma patients with a head AIS $>2$ and at least one other body system with an AIS $>2$ in order to assess the impact of isolated TBI, rather than concomitant injuries, on the development of coagulopathy.

Although our comparison group had a head AIS $<2$, an AIS $>2$ in one or more other body regions may have been present. These other systemic injuries may have contributed to ACoT as approximately $25 \%$ of all seriously injured trauma patients have a coagulopathy [31,42]. The etiology of ACoT is complex and not completely understood, and therefore many factors including the release of tissue factor, consumption of platelets and coagulation factors, hemodilution, acidosis, hypothermia, transfusions, and hyperfibrinolysis may have been present in either group [43]. As many of these same mechanisms are present in TBI, it is difficult to distinguish or verify if the effects are separate, co-existing, or confounding. Finally, patients that were on antiplatelet or anticoagulant therapies prior to admission were not identified and therefore, we did not account for pharmaceutically induced coagulopathy within either group of patients.

\section{Conclusions}

No difference in the prevalence of coagulopathy between TBI and non-TBI patients was detected by CCT values. However, admission r-TEG data demonstrated a higher prevalence of coagulopathy in TBI patients, and both $\mathrm{mA}<55$ and alpha-angle $<56$ are associated with increased 24-hour and 30 day mortality. Translation of this information into the clinical practice may include platelet and cryoprecipitate transfusions or administration of fibrinogen concentrates based on abnormal r-TEG values. Further studies are necessary to validate our findings, as well as to investigate whether administration of these products can correct coagulopathy and improve TBI outcomes.

\section{Funding/Disclosures}

The NIGMS T32GM008792 (JCC), The State of Texas Emerging Technology Fund (CEW, JBH, and BAC) and the University of Texas Health Science Center's Center provided support for this work for Translational Injury Research.

\section{Acknowledgements}

The authors would like to thank Angela Beeler for her editorial support.

\section{References}

1. Acosta JA, Yang JC, Winchell RJ, Simons RK, Fortlage DA, et al. (1998) Lethal injuries and time to death in a level I trauma center. J Am Coll Surg 186: 528533.

2. Shackford SR, Mackersie RC, Holbrook TL, Davis JW, Hollingsworth-Fridlund $\mathrm{P}$, et al. (1993) The epidemiology of traumatic death. A population-based analysis. Arch Surg 128: 571-575.

3. Lustenberger T, Talving P, Kobayashi L, Inaba K, Lam L, et al. (2010) Time course of coagulopathy in isolated severe traumatic brain injury. Injury 41: 924 928.

4. Talving P, Benfield R, Hadjizacharia P, Inaba K, Chan LS, et al. (2009) Coagulopathy in severe traumatic brain injury: a prospective study. J Trauma 66: 55-61.

5. Harhangi BS, Kompanje EJ, Leebeek FW, Maas Al (2008) Coagulation disorders after traumatic brain injury. Acta Neurochir (Wien) 150: 165-175.

6. Cap AP, Spinella PC (2011) Severity of head injury is associated with increased risk of coagulopathy in combat casualties. J Trauma 71: S78-81.

7. Wafaisade A, Lefering R, Tjardes T, Wutzler S, Simanski C, et al. (2010) Acute coagulopathy in isolated blunt traumatic brain injury. Neurocrit Care 12: 211 219

8. Kunio NR, Differding JA, Watson KM, Stucke RS, Schreiber MA (2012) Thrombelastography-identified coagulopathy is associated with increased morbidity and mortality after traumatic brain injury. American journal of surgery. 203: 584-588.

9. Windelov NA, Welling KL, Ostrowski SR, Johansson PI (2011) The prognostic value of thrombelastography in identifying neurosurgical patients with worse prognosis. Blood coagulation \& fibrinolysis: an international journal in haemostasis and thrombosis 22: 416-419.

10. Zehtabchi S, Soghoian S, Liu Y, Carmody K, Shah L, et al. (2008) The association of coagulopathy and traumatic brain injury in patients with isolated head injury. Resuscitation 76: 52-56.

11. Salehpour F, Bazzazi AM, Porhomayon J, Nader ND (2011) Correlation between coagulopathy and outcome in severe head trauma in neurointensive care and trauma units. J Crit Care 26: 352-356.

12. Nekludov M, Bellander BM, Blombäck M, Wallen HN (2007) Platelet dysfunction in patients with severe traumatic brain injury. J Neurotrauma 24: 1699-1706.

13. Kitchens CS (2005) To bleed or not to bleed? Is that the question for the PTT? J Thromb Haemost 3: 2607-2611.

14. Dzik WH (2004) Predicting hemorrhage using preoperative coagulation screening assays. Curr Hematol Rep 3: 324-330.

15. Jeger V, Zimmermann H, Exadaktylos AK (2009) Can RapidTEG accelerate the search for coagulopathies in the patient with multiple injuries? J Trauma 66: 1253-1257.

16. Cotton BA, Faz G, Hatch QM, Radwan ZA, Podbielski J, et al. (2011) Rapid thrombelastography delivers real-time results that predict transfusion within 1 hour of admission. J Trauma 71: 407-414.

17. De Nicola P, Mazzetti GM (1955) Evaluation of thrombelastography. Am J Clin Pathol 23: 447-452.

18. Johansson PI (2012) Coagulation monitoring of the bleeding traumatized patient. Curr Opin Anaesthesiol 25: 235-241.

19. Martini WZ, Cortez DS, Dubick MA, Park MS, Holcomb JB (2008) Thrombelastography is better than PT, aPTT, and activated clotting time in detecting clinically relevant clotting abnormalities after hypothermia hemorrhagic shock and resuscitation in pigs. The Journal of trauma 65: 535543

20. Plotkin AJ, Wade CE, Jenkins $\mathrm{DH}$ (2008) A reduction in clot formation rate and strength assessed by thrombelastography is indicative of transfusion requirements in patients with penetrating injuries. The Journal of trauma 64 S64-68.

21. Holcomb JB, Minei KM, Scerbo ML (2012) Admission rapid thrombelastography can replace conventional coagulation tests in the emergency department: experience with 1974 consecutive trauma patients. Annals of surgery 256: 476486. 
Citation: Sixta SL, Cardenas JC, Kitagawa R, Wade CE, Holcomb JB, et al. (2015) Hypocoagulability in Traumatic Brain Injury as Measured by Traditional Means and Thrombelastography. J Neurol Neurophysiol 6: 316. doi:10.4172/2155-9562.1000316

22. Hosmer DW LS (2000) Applied Logistic Regression. Second ed. New York, NY: Wiley.

23. Stein SC, Smith DH (2004) Coagulopathy in traumatic brain injury. Neurocrit Care 1: 479-488.

24. Halpern CH, Reilly PM, Turtz AR, Stein SC (2008) Traumatic coagulopathy: the effect of brain injury. J Neurotrauma 25: 997-1001.

25. Laroche M, Kutcher ME, Huang MC, Cohen MJ, Manley GT (2012) Coagulopathy after traumatic brain injury. Neurosurgery 70: 1334-1345.

26. Nemerson Y, Giesen PL (1998) Some thoughts about localization and expression of tissue factor. Blood Coagul Fibrinolysis 9 Suppl 1: S45-47.

27. Carrick MM, Tyroch AH, Youens CA, Handley T (2005) Subsequent development of thrombocytopenia and coagulopathy in moderate and severe head injury: support for serial laboratory examination. J Trauma 58: 725-729.

28. Schnüriger B, Inaba K, Abdelsayed GA, Lustenberger T, Eberle BM, et al. (2010) The impact of platelets on the progression of traumatic intracranial hemorrhage. J Trauma 68: 881-885.

29. Brohi K, Cohen MJ, Davenport RA (2007) Acute coagulopathy of trauma: mechanism, identification and effect. Curr Opin Crit Care 13: 680-685.

30. Cohen MJ, Brohi K, Ganter MT, Manley GT, Mackersie RC et al. (2007) Early coagulopathy after traumatic brain injury: the role of hypoperfusion and the protein C pathway. The Journal of trauma. 63:1254-1261; discussion 12611252.

31. Brohi K, Singh J, Heron M, Coats T (2003) Acute traumatic coagulopathy. J Trauma 54: 1127-1130.

32. Allard CB, Scarpelini S, Rhind SG, Baker AJ, Shek PN, et al. (2009) Abnormal coagulation tests are associated with progression of traumatic intracranial hemorrhage. J Trauma 67: 959-967.

33. Stein SC, Young GS, Talucci RC, Greenbaum BH, Ross SE (1992) Delayed brain injury after head trauma: significance of coagulopathy. Neurosurgery 30 : 160-165.
34. Selladurai BM, Vickneswaran M, Duraisamy S, Atan M (1997) Coagulopathy in acute head injury--a study of its role as a prognostic indicator. $\mathrm{Br} \mathrm{J}$ Neurosurg 11: 398-404.

35. Olson JD, Kaufman HH, Moake J, O'Gorman TW, Hoots K, et al. (1989) The incidence and significance of hemostatic abnormalities in patients with head injuries. Neurosurgery 24: 825-832.

36. Murray GD, Butcher I, McHugh GS, Lu J, Mushkudiani NA, et al. (2007) Multivariable prognostic analysis in traumatic brain injury: results from the IMPACT study. J Neurotrauma 24: 329-337.

37. Davis PK, Musunuru H, Walsh M, Cassady R, Yount R, et al. (2013) Platelet dysfunction is an early marker for traumatic brain injury-induced coagulopathy. Neurocrit Care 18: 201-208.

38. Carroll RC, Craft RM, Langdon RJ (2009) Early evaluation of acute traumatic coagulopathy by thrombelastography. Translational research: the journal of laboratory and clinical medicine 154: 34-39.

39. Wade CE, Dubick MA, Blackbourne LH, Holcomb JB (2009) It is time to assess the utility of thrombelastography in the administration of blood products to the patient with traumatic injuries. J Trauma 66: 1258.

40. Reikvam H, Steien E, Hauge B (2009) Thrombelastography. Transfusion and apheresis science: official journal of the World Apheresis Association : official journal of the European Society for Haemapheresis 40: 119-123.

41. Tauber H, Innerhofer P, Breitkopf R, Westermann I, Beer R, et al. (2011) Prevalence and impact of abnormal ROTEM $(R)$ assays in severe blunt trauma: results of the 'Diagnosis and Treatment of Trauma-Induced Coagulopathy (DIATRE-TIC) study'. Br J Anaesth 107: 378-387.

42. Niles SE, McLaughlin DF, Perkins JG, Wade CE, Li Y, et al. (2008) Increased mortality associated with the early coagulopathy of trauma in combat casualties. J Trauma 64: 1459-1463.

43. Hess JR, Brohi K, Dutton RP, Hauser CJ, Holcomb JB, et al. (2008) The coagulopathy of trauma: a review of mechanisms. J Trauma 65: 748-754. 